\title{
Oral antibiotic therapy in diabetic foot osteomyelitis: one small step or a giant leap of faith?
}

\author{
Prashanth R. J. Vas ${ }^{1}$, Maria Demetriou ${ }^{2}$, Nikolaos Papanas ${ }^{2}$ \\ ${ }^{1}$ Diabetic Foot Clinic, King's College Hospital, London, UK; ${ }^{2}$ Diabetes Centre-Diabetic Foot Clinic, Second Department of Internal Medicine, \\ Democritus University of Thrace, Alexandroupolis, Greece \\ Correspondence to: Nikolaos Papanas. Diabetic Foot Clinic, Diabetes Centre, Second Department of Internal Medicine, Democritus University of \\ Thrace, G. Kondyli 22c, Alexandroupolis 68100, Greece. Email: papanasnikos@yahoo.gr. \\ Provenance: This is an invited article commissioned by the Editorial Office, Annals of Translational Medicine. \\ Comment on: Li HK, Rombach I, Zambellas R, et al. Oral versus Intravenous Antibiotics for Bone and Joint Infection. N Engl J Med 2019;380:425-36.
}

Submitted Nov 03, 2019. Accepted for publication Dec 02, 2019.

doi: $10.21037 / \mathrm{atm} .2019 .12 .53$

View this article at: http://dx.doi.org/10.21037/atm.2019.12.53

One in 3 subjects with diabetes may experience foot ulcers during their lifetime. Over half of such ulcerations are complicated by infection (1), often involving bones as well. It is estimated that a degree of diabetic foot osteomyelitis (DFO) may affect $20-60 \%$ of all infected diabetic foot ulcers (2). The presence of DFO is associated with significantly poor clinical outcomes, including slower time to healing, longer duration of hospitalisation and a higher likelihood of amputation (3). The presentation of DFO can often be insidious. Indeed, the typical features of axial bone and joint infections, such as pain and localised erythema, can often be absent or subtle. Thus, detection and management of DFO remains one of the most challenging and debated facets of diabetic foot care $(2,3)$.

Important treatment opportunities in DFO have evolved over the last few decades. Previously, surgical treatment was often the only available choice, mainly involving amputation to a point proximal to the DFO. However, over the last 2 decades, it has been established that non-surgical treatment of DFO is frequently feasible, particularly in those presenting without limb threatening infection (4). Available data supports the notion that DFO remission rates between $64-82 \%$ may be achieved in carefully selected patients receiving conservative medical treatment without surgery (5-9).

Successful non-surgical management of DFO is underpinned by the use of antibiotic therapy to suppress infection and to achieve bone sterility. In their series of DFO treated without surgery, Senneville et al. (5) have reported that antibiotic therapy based on bone cultures was the only variable associated with remission in a multivariate analysis (odds ratio 4.78; 95\% confidence interval (CI): 1.0-22.7, $\mathrm{P}=0.04)$. However, a significant number of controversies remain on the use of antibiotics in DFO. Antibiotic selection can be notoriously complex and may depend on how carefully the specimen was obtained, sensitivities declared by the microbiology laboratory, and often, the personal preferences of the clinician. Duration of antibiotic treatment is also unclear (5-7). Often extended antibiotic therapy regimes are utilised. Game and Jeffcoate (6) have reported an apparent remission rate of $83 \%$ with antibiotics alone, with a median length of initial antibiotic treatment amounting to 61 days (range, 3-349 days). However, in the only published randomised controlled trial on this subject (9), a 6-week antibiotic regime was not significantly inferior to a 12 -week regime $(60 \%$ at 6 weeks vs. $70 \%$ at 12 weeks, $\mathrm{P}=0.50)$. Those receiving 12 weeks antibiotics had a higher rate of adverse events related to the use of antibiotics (9).

Perhaps the most central debate is the optimal mode of antibiotic delivery. The use of long-term intravenous antibiotic therapy was embraced in DFO treatment, as a concept borrowed from axial bone and joint infections (7). This meant that many individuals with DFO received long duration intravenous antibiotic therapy, often lasting weeks and anecdotally for many months. Such regimes came with their inherent challenges of safe maintenance of long-term intravenous lines, rigorous of daily antibiotic administration, 
and importantly, the systemic adverse effects that would be expected with such treatment (8), not to mention the risk of antimicrobial resistance and cost $(7,8)$. Although many published studies on achieving remission of DFO through non-surgical means have utilised oral treatment regimens preceded by a short duration of intravenous therapy $(6,9,10)$ and even recommended in major DFO guidelines $(10,11)$, there has been a lack of randomised trial evidence to support the notion that oral antibiotic therapy is noninferior to intravenous therapy in achieving remission, not only in DFO but also bone and joint infections in general.

The OVIVA (oral versus intravenous antibiotics for bone and joint infection) trial was a multicentre randomised controlled trial involving 1,054 patients from the United Kingdom, comparing 1 year outcomes between early intravenous-to-oral antibiotic switch (less than 7 days) with continued intravenous therapy for at least 6 weeks in adults with bone and joint infection followed up for 1 year $(12,13)$. Lower limb infections were the predominant cohort $(82.7 \%)$ and $16.5 \%$ overall had foot infections. The primary outcome was definite treatment failure: this was noted in $14.6 \%$ of participants in the intravenous group and $13.2 \%$ of the oral switch group (-1.4 percentage points; $95 \%$ CI: -5.6 to $2.9, \mathrm{P}=$ non-significant), thereby indicating noninferiority (12). Similarly, there was no between-group difference in probable or possible treatment failure rates (12). Interestingly, and highlighting the challenges with long duration intravenous antibiotic therapy, there was a higher early discontinuation rate of initial assigned therapy in the intravenous group than in the oral group (18.9\% vs. $12.8 \%$, $\mathrm{P}=0.006)$ (12). In addition, oral antibiotic switch therapy was associated with a shorter length of hospital stay and fewer complications related to catheter use than intravenous therapy (13).

Certainly, such a large and complex trial had a few key limitations $(12,13)$. If there was an isolate without an oral substitute or if subacute bacterial endocarditis was coexistent, patients were excluded. Furthermore, patient selection, whilst being relevant to the daily clinical practice for infectious disease teams working within large hospitals, was limited to bone and joint infections presenting with localised pain, localised erythema with temperature $>38.0^{\circ} \mathrm{C}$ or a discharging sinus/wound. Finally, the study was not powered for subgroup analyses $(12,13)$.

What, then, can we learn from the OVIVA study in the field of DFO? First, it is important to recognise that it is unclear how many of the $19.5 \%$ with diabetes were included for active DFO treatment. Therefore, immediate generalisability of these findings to DFO treatment cannot be made with absolute confidence $(12,13)$. Secondly, DFO is almost invariably complicated by ongoing ulceration. Given the often evolving microbiological milieu, this renders interpretation of culture results very challenging and dubious. Multidrug resistance is also emerging as a crucial problem, and we should not forget that the OVIVA trial excluded such patients $(12,13)$. Finally, the study was carried out by expert multidisciplinary teams, and so results cannot easily be extrapolated to less expert settings.

Managing DFO is not simply about choosing between a surgical or non-surgical route (12-14). Conversely, it is important to ensure the following issues: (I) all infected but non-viable necrotic bone must be removed when necessary; (II) there must be adequate tissue perfusion and any significant peripheral arterial disease has been corrected; (III) multidisciplinary expertise is maintained, including offloading and preventing new infection; and (IV) appropriate antibiotics must be used (10-15).

In conclusion, the OVIVA trial is an important step in our understanding of antimicrobial therapy $(12,13)$. It has shown for the first time, in a randomised controlled setting, that an early oral antibiotic switch is not inferior to long courses of intravenous antibiotics. However, one important limitation is the inability to confirm benefit by subgroup analysis, including DFO. Hence, further experience is clearly welcome.

\section{Acknowledgments}

None.

\section{Footnote}

Conflicts of Interest: PRJ Vas has received speaker honoraria from Sanofi Diabetes and MSD. N Papanas has been an advisory board member of TrigoCare International, Abbott, AstraZeneca, Elpen, MSD, Novartis, Novo Nordisk, SanofiAventis and Takeda; has participated in sponsored studies by Eli Lilly, MSD, Novo Nordisk, Novartis and SanofiAventis; received honoraria as a speaker for AstraZeneca, Boehringer Ingelheim, Eli Lilly, Elpen, Galenica, MSD, Mylan, Novartis, Novo Nordisk, Pfizer, Sanofi-Aventis, Takeda and Vianex; and attended conferences sponsored by TrigoCare International, AstraZeneca, Boehringer Ingelheim, Eli Lilly, Novartis, Novo Nordisk, Pfizer and Sanofi-Aventis. M Demetriou has no conflicts of interest to declare. 
Ethical Statement: The authors are accountable for all aspects of the work in ensuring that questions related to the accuracy or integrity of any part of the work are appropriately investigated and resolved.

\section{References}

1. Ndosi M, Wright-Hughes A, Brown S, et al. Prognosis of the infected diabetic foot ulcer: a 12-month prospective observational study. Diabet Med 2018;35:78-88.

2. Shone A, Burnside J, Chipchase S, et al. Probing the validity of the probe-to-bone test in the diagnosis of osteomyelitis of the foot in diabetes. Diabetes Care 2006;29:945.

3. Wukich DK, Hobizal KB, Sambenedetto TL, et al. Outcomes of Osteomyelitis in Patients Hospitalized With Diabetic Foot Infections. Foot Ankle Int 2016;37:1285-91.

4. Lázaro Martínez JL, García Álvarez Y, TardáguilaGarcía A, et al. Optimal management of diabetic foot osteomyelitis: challenges and solutions. Diabetes Metab Syndr Obes 2019;12:947-59.

5. Senneville E, Lombart A, Beltrand E, et al. Outcome of diabetic foot osteomyelitis treated nonsurgically: a retrospective cohort study. Diabetes Care 2008;31:637-42.

6. Game FL, Jeffcoate WJ. Primarily non-surgical management of osteomyelitis of the foot in diabetes. Diabetologia 2008;51:962-7.

7. Wagner DK, Collier BD, Rytel MW. Long-term intravenous antibiotic therapy in chronic osteomyelitis. Arch Intern Med 1985;145:1073-8.

Cite this article as: Vas PRJ, Demetriou M, Papanas N. Oral antibiotic therapy in diabetic foot osteomyelitis: one small step or a giant leap of faith? Ann Transl Med 2019;7(Suppl 8):S266. doi: $10.21037 / \mathrm{atm} .2019 .12 .53$
8. van Asten SAV, Mithani M, Peters EJG, et al. Complications during the treatment of diabetic foot osteomyelitis. Diabetes Res Clin Pract 2018;135:58-64.

9. Tone A, Nguyen S, Devemy F, et al. Six-week versus twelve-week antibiotic therapy for nonsurgically treated diabetic foot osteomyelitis: a multicenter openlabel controlled randomized study. Diabetes Care 2015;38:302-7.

10. Lipsky BA, Aragon-Sanchez J, Diggle M, et al. IWGDF guidance on the diagnosis and management of foot infections in persons with diabetes. Diabetes Metab Res Rev 2016;32 Suppl 1:45-74.

11. Lipsky BA, Berendt AR, Cornia PB, et al. 2012 Infectious Diseases Society of America clinical practice guideline for the diagnosis and treatment of diabetic foot infections. Clin Infect Dis 2012;54:e132-73.

12. Li HK, Rombach I, Zambellas R, et al. Oral versus Intravenous Antibiotics for Bone and Joint Infection. N Engl J Med 2019;380:425-36.

13. Scarborough M, Li HK, Rombach I, et al. Oral versus intravenous antibiotics for bone and joint infections: the OVIVA non-inferiority RCT. Health Technol Assess 2019;23:1-92.

14. Panagopoulos P, Drosos G, Maltezos E, et al. Local antibiotic delivery systems in diabetic foot osteomyelitis: time for one step beyond? Int J Low Extrem Wounds 2015;14:87-91.

15. Vas PRJ, Panagopoulos P, Papanas N. Diabetic foot fungal osteomyelitis: No longer unknown and hidden? Int J Low Extrem Wounds 2018;17:142-3. 